\title{
EVALUATION OF SMOKING EFFECT ON TELOMERE LENGTH OF THE CHROMOSOMES OF ALZHEIMER'S PATIENTS
}

\author{
Engy S. Sayed ${ }^{(1)}$; Mostafa H. Ragab ${ }^{(2)}$; Hamdy H. Souilm ${ }^{(3)}$ \\ Marwa I. Shehab ${ }^{(1)}$; Somaia M. Ismail ${ }^{(1)}$; Maissa E. Fif $\mathbf{y}^{(4)}$ \\ Azzah A. Khedr( ${ }^{(1)}$ and Azzah E. Abdel Naby ${ }^{(1)}$
}

1) National Research Centre 2) Institute of Environmental Studies and Research, Ain Shams University 3) Faculty of Science, Ain Shams University 4) Okasha Institute of psychiatry, Ain Shams University

\begin{abstract}
Background: Alzheimer's disease (AD) is a common and severe neurodegenerative disorder. Human chromosomes telomeres are essential for the maintenance of genomic stability and play prominent roles in both cellular senescence and ageing of the organisms. Regulation of telomere length (TL) is a result of complex interaction between environmental and genetic factors.

Aim: measuring the length of telomere length in AD patients by Quantitative Florescence in Situ Hybridization (Q-FISH) methodology and studying of smoking as an environmental risk factor in the development of Alzheimer's disease.

Subjects and Methods: This study included $10 \mathrm{AD}$ patients and 10 controls. All blood samples were collected measured by using Q-FISH to assess the telomere length.

Results: It was found a significant difference in TL between AD patients and the controls, with a standardized mean difference resulted $p=0.03$ were considered statistically significant which is still significant $p<0.05$. Smokers had shorter telomeres length in $\mathrm{AD}$ patients than non-smokers.

Conclusion: A significant correlation was found between AD and telomere length shortening. The environmental influence of smoking had an additional effect on telomere shortening in AD.
\end{abstract}


Key words: Alzheimer's disease, telomere length, Q-FISH, smoking, environmental influence.

\section{INTRODUCTION}

The human brain is a remarkable organ that allows us to carry out every element of our daily lives. It manages many body functions, such as breathing, blood circulation, and digestion, without our prior knowledge or conscious direction. The brain embodies nerve cells and supportive glial cells, which hold the nerve cells in place and provide them with nutrients (Pray et al., 2015).

Alzheimer's disease is a neurological heterogeneous genetic disorder in which death of brain cells causes neurodegenerative symptoms such as memory loss and cognitive decline. The neurodegenerative symptoms of Alzheimer's disease gradually worsen overtime with progressive shrinkage of the total brain size and decline of the number of nerve cells and their connections (Barber, 2012) .

The brains of patients with Alzheimer's disease have abnormal clumps of cellular debris, proteins (plaques) and collapsed microtubules, which normally support the structures inside the brain cells. Microtubule collapse is caused by a malfunctioning protein called tau. In Alzheimer's patients, tau proteins cluster together to form disabling plaques and tangles, which damage the healthy cells around them, leading to cell death (Serrano-pozo et al., 2011). 
Journal of Environmental Sciences (JES)

Institute of Environmental Studies and Research, Ain Shams University

Sayed, Ingy et al.

There are two main types of $\mathrm{AD}$, the rare early-onset Alzheimer's disease which usually affects people aged 30 to 60 . Some cases of early-onset disease are inherited and are called familial $\mathrm{AD}(\mathrm{FAD})$. The other type is late-onset Alzheimer's disease. It is the more common form and occurs in those of 60 years and older. Gaining insight into the genetic factors associated with both forms of $\mathrm{AD}$ is important because identifying genes that either cause the disease or influence a person's risk of developing it improves our ability to understand how and why the disease starts and progresses (Bird, 2009) .

The most common form of the disease_which usually strikes after the age of 65 years_ is likely linked to the Apolipoprotein E (ApoE) gene on chromosome 19q13.2 (Bertram, 2012). Mutations in genes found on chromosomes 1, 14, and 21 are linked to rarer forms of the disease, which strike earlier in life (Wu et al., 2012).

Scientists do not know how ApoE gene, which has three forms, affects the development of Alzheimer's disease. The presence of one form (ApoE4) does increase a person's risk of developing Alzheimer's disease, however, the other two forms seem to protect against the disease. Although people who inherit the ApoE4 form of the gene are at an increased risk for getting Alzheimer's disease; they will not necessarily develop it (Ungar et al., 2014).

Alzheimer disease is a genetically heterogeneous disorder where AD2 (OMIM 104310), associated with the ApoE4 allele (107741) on chromosome 19; AD3 (OMIM 607822), caused by mutation in the presenilin-1 gene 
(PSEN1; OMIM 104311) on 14q and AD4 (OMIM 606889), is caused by a mutation in the PSEN2 gene (OMIM 600759) on 1q31 (Corder et al., 1993).

There are several specific environmental risk factors in the development of dementia and Alzheimer's disease. Dietary risk factors and nutrition are emerging as critical factors in brain health (Crous-bou et al., 2017).

Telomeres are repeating DNA hexamer (TTAGGG) sequences found at the ends of chromosomes. Telomeres are important for replication of the chromosomes during cell division. In each cell division, telomeres are shortened. When telomeres become critically short, chromosomes can undergo end fusions, aberrant recombination, and degradation. Thus, maintenance of sufficient telomere length plays a major role in chromosomal stability and cell protection (Muraki et al., 2012) .

Short telomeres were associated with ageing and the development of age _related diseases. Free radicals and oxidative stress have been suggested, for a long time, to be involved in the ageing process. Von Zglinicki and colleagues argue that telomere length is a marker for a person's ability to withstand DNA damage reflected by aging and oxidative stress (Passos, Saretzki and Zglinicki, 2007). In light of Von Zglinicki view, it seems that life extension may be more dependent on comprehensive antioxidant protection (Reichert and Reichert, 2014). The Scientists suggested that it would be valuable to monitor the cumulative effect of long-term use of antioxidant on telomere length and correlating that to general health status (Heidinger et al., 2012; Shalev, 2012). 
Environmental toxins such as air pollution have been also implicated in Alzheimer's disease risk. Exposure to air pollution can lead to chronic oxidative stress, which is involved in the pathogenesis of Alzheimer's disease (Moulton and Yang, 2012).

Smoking increases the severity of abnormalities typical of Alzheimer's disease, including amyloid genesis (the production of amyloid), neuro inflammation and tau phosphorylation (this causes tangles within nerve cells). Cigarette smoking may therefore both hasten the onset of Alzheimer's disease and exacerbate its features.

Cigarette smoking results in exposure to free radicals and reactive oxygen species, which leads to increased oxidative stress and inflammation. Both processes have been also linked to TL shortening, leading to the hypothesis that smoking promotes TL shortening (Van der Vaart et al., 2004).

Traditionally, the mean telomere length was measured by Southern Blot analysis of terminal restriction fragments (TRFs), obtained by digesting the DNA with certain restriction enzymes that do not cut through telomere repeats (Jenkins et al., 2017).

Individual telomeres of certain chromosomes can be measured by; Single Telomere Elongation Length Analysis (STELA) (Lai et al., 2018).

Another method has been used to measure telomere length by the use of Peptide Nucleic Acid (PNA) probes. Quantitative Florescence in Situ Hybridization (Q-FISH) allows us to visualize telomeres during metaphase, where the staining of telomeres with a PNA probe is proportionate to their 
sizes, which permits the comparison of telomeres' lengths between different chromosomes (Perner et al., 2003). .

Quantitative Florescence in Situ Hybridization (Q-FISH) is an approach combining fluorescence in situ hybridization (FISH) and digital quantification of microscopic images. It has been shown to be applicable for a variety of purposes in molecular cytogenetic studies. Q FISH represents an important part of studying somatic chromosomal mosaicism and molecular cytogenetic detection of chromosomal variations in interphase nuclei (Iourov, 2017).

The objective of this study is to measure the length of telomere in Alzheimer's patients by Quantitative Florescence in Situ Hybridization (QFISH) methodology and investigate the probable relation.

\section{MATERIALS AND METHODS}

Materials: The study included 10 Egyptian Alzheimer's disease patients (3 males and 7 females), with age range 55 -80 years, and 10 control volunteers with matched age and sex. Our control was from the National Research Centre, Cairo, Egypt.

The patients were assigned according to detailed patient history, physical and neurological exams and lab tests, neuropsychological tests, imaging tools such as "CT" scan, or magnetic resonance imaging (MRI) and positron emission tomography (PET) scans. Diagnoses have been made by a specialist from Geriatric Psychiatry Clinics, Faculty of Medicine, Ain Shams University. The approval of the study was processed by the Ethical 
Committee and informed written consent was obtained from all the participants.

Methods: All patients were subjected to:

1) Clinical investigation:

- The family history of the patients was taken with laying stress on neuropsychological examination.

- Questionnaires were done for Alzheimer's patient's guardians designed to detect some environmental risk factors in their life style and education (Bertram et al., 2007) .

2) Cytogenetic evaluation: Peripheral blood was collected on heparin and cultured according to "Verma and Babu, 1995". This samples were assessed by Quantitative Fluorescence in-situ Hybridization (Q-FISH) technique,

- Metaphase-arrested the cells were prepared; spreading of the cells was carried out by splashing on slides.

- The slides were fixed in formaldehyde and treated with pepsin.

- PNA probe was denaturized by heating and hybridized on prepared slide.

- The slides were washed to remove unbound PNAs and left over night for aging. DAPI or PI counterstain was added and left for at least 15 mins.

- Image was captured, and analysis was performed by using software according to "Perner et al., 2003". 


\section{Statistical analysis of T/C data:}

Analysis of telomere length: From each of the donors ten metaphases were captured with a fluorescence microscope (Axioscope 2; Zeiss, Jena, Germany), which is equipped with a CCD camera (resolution 752 ! 576 pixels, 11 _ $\mathrm{m}$ pixel size, on-chip integration) and linked to the ISIS software (MetaSystems, Altlussheim, Germany).

Normalized data were derived by calculating the ratio of the absolute telomere intensities and the reference signal intensities (centromere intensities) of each metaphase (T/C value) (Perner et al., 2003).Statistical tests were performed using T-test.

\section{RESULTS}

The age of $\mathrm{AD}$ patients ranged 51 to 68 years, mean $\pm \mathrm{SD}(59.4 \pm 6.02)$. The main clinical and functional characteristics data of the AD patients and the control studied are shown in "Table 1". The AD patients have gender (male 30\%_female 70\%), education (no 30\% _ yes 70\%) and smoking (no $50 \%$ _ yes $50 \%$ ).

The primary data were afterwards compiled by the software and resulted in mean telomere intensities of the chromosomes for every metaphase. The mean telomere intensity for each chromosome, standard deviations, median and the interquartile range were automated calculates.

The means were used for statistical analysis. Ten metaphases from each individual were examined (Fig .1) and the median of the T/C-FISH value was 42 
calculated. The equation of the resulting regression line was $y=2507+204 x$, with $x$ representing the T/C value and y the corresponding TRF value in base pairs. In accord with this calibration, TRF values were derived from T/C data (Perner et al., 2003).

A significant difference in TL between the AD patients and the controls, with a standardized mean difference resulted $\mathrm{p}=0.03$ were considered statistically significance $\mathrm{p}<0.05$.

This study found that an increase in the years of those been affected by $\mathrm{AD}$ is significantly correlated with decreases in telomere length in all the $\mathrm{AD}$ patients.

Results of mean and SD telomere length and age of the normal controls and the AD patients are investigated and shown in Table 2.

Smokers had shorter telomeres length in the AD patients than the nonsmokers. The results show that the mean \pm Sd of the smokers $(4.88 \pm 1.96)$ is less than mean $\pm \mathrm{Sd}(5.72 \pm 2.13)$ of the non-smokers. 
Sayed, Ingy et al.

Table (1): Clinical characteristics of controls and cases of the AD patients

\begin{tabular}{|c|c|c|c|}
\hline & Parameter & Control (n=10) & Cases (n=10) \\
\hline \hline \multirow{2}{*}{ Age } & Mean \pm Sd & $59.4 \pm 6.02$ & $71.7 \pm 11.06$ \\
& Range years & $(51-68) \mathrm{y}$ & $(56-90)$ \\
\hline \multirow{2}{*}{ Gender } & Male & M 4 (40\%) & M 3 (30\%) \\
& Female & F 6 (60\%) & F 7 (70\%) \\
\hline \multirow{2}{*}{ Marital status } & Married & Married 8 (80\%) & Married 4 (40\%) \\
& Widowed & Widowed 2 (20\%) & Widowed 6 (60\%) \\
\hline \multirow{2}{*}{ Education } & Yes & Yes 9 (90\%) & Yes 3 (30\%) \\
& No & No 1 (10\%) & No 7 (70\%) \\
\hline \multirow{2}{*}{ Smoking } & Yes & Yes 2 (20\%) & Yes 5 (50\%) \\
& No & No 8 (80\%) & No 5 (50\%) \\
\hline \multirow{2}{*}{ Living status } & Alone & $1(10 \%)$ & $3(30 \%)$ \\
& With others & $9(90 \%)$ & $7(70 \%)$ \\
\hline \multirow{2}{*}{ Hypertension } & Normal & $4(40 \%)$ & $1(10 \%)$ \\
& Controlled & $5(50 \%)$ & $8(80 \%)$ \\
& Uncontrolled & $1(10 \%)$ & $1(10 \%)$ \\
\hline \multirow{2}{*}{ Diabetes } & Normal & $5(50 \%)$ & $1(10 \%)$ \\
& Controlled & $4(40 \%)$ & $7(70 \%)$ \\
& Uncontrolled & $1(10 \%)$ & $2(20 \%)$ \\
\hline Family history of & Negative & $9(90 \%)$ & $6(60 \%)$ \\
Alzheimer's & Positive & $1(10 \%)$ & $4(40 \%)$ \\
\hline \hline
\end{tabular}


Table (2): Mean and SD telomere length and age of normal controls and AD patients

\begin{tabular}{|c|c|c|}
\hline NO & $\begin{array}{l}\text { TL Mean (\%) } \pm \text { (SD of } \\
\text { Telomeres length) } \\
\text { (kb) of the AD patients }\end{array}$ & $\begin{array}{l}\text { TL Mean (\%) } \pm \text { (SD of } \\
\text { Telomeres length) } \\
(k b) \text { of the control }\end{array}$ \\
\hline 1. & $7,00 \pm 6.3$ & $41.17 \pm 25.34$ \\
\hline 2. & $1 \cdot, \wedge \pm 9.08$ & $10.5 \pm 9.8$ \\
\hline 3. & $7,00 \pm 6.3$ & $49.0 \pm 45.2$ \\
\hline 4. & $r \neg, \vee \pm 63.75$ & $25.05 \pm 24.3$ \\
\hline 5. & $\curlyvee, \lessdot \wedge \pm 9.17$ & $9.68 \pm 8.7$ \\
\hline 6. & $\vee, 7 \pm 7.62$ & $17.5 \pm 19.3$ \\
\hline 7. & $r \vee, T V \pm 91.9$ & $33.8 \pm 31.6$ \\
\hline 8. & $2.38 \pm 3.2$ & $26.2 \pm 24.9$ \\
\hline 9. & $15.74 \pm 14.2$ & $12.7 \pm 13.5$ \\
\hline 10. & $23.05 \pm 25.3$ & $43.2 \pm 41.7$ \\
\hline Mean $(\%) \pm$ SD & $13.34 \pm 9.33$ & $26.88 \pm 14.39$ \\
\hline
\end{tabular}




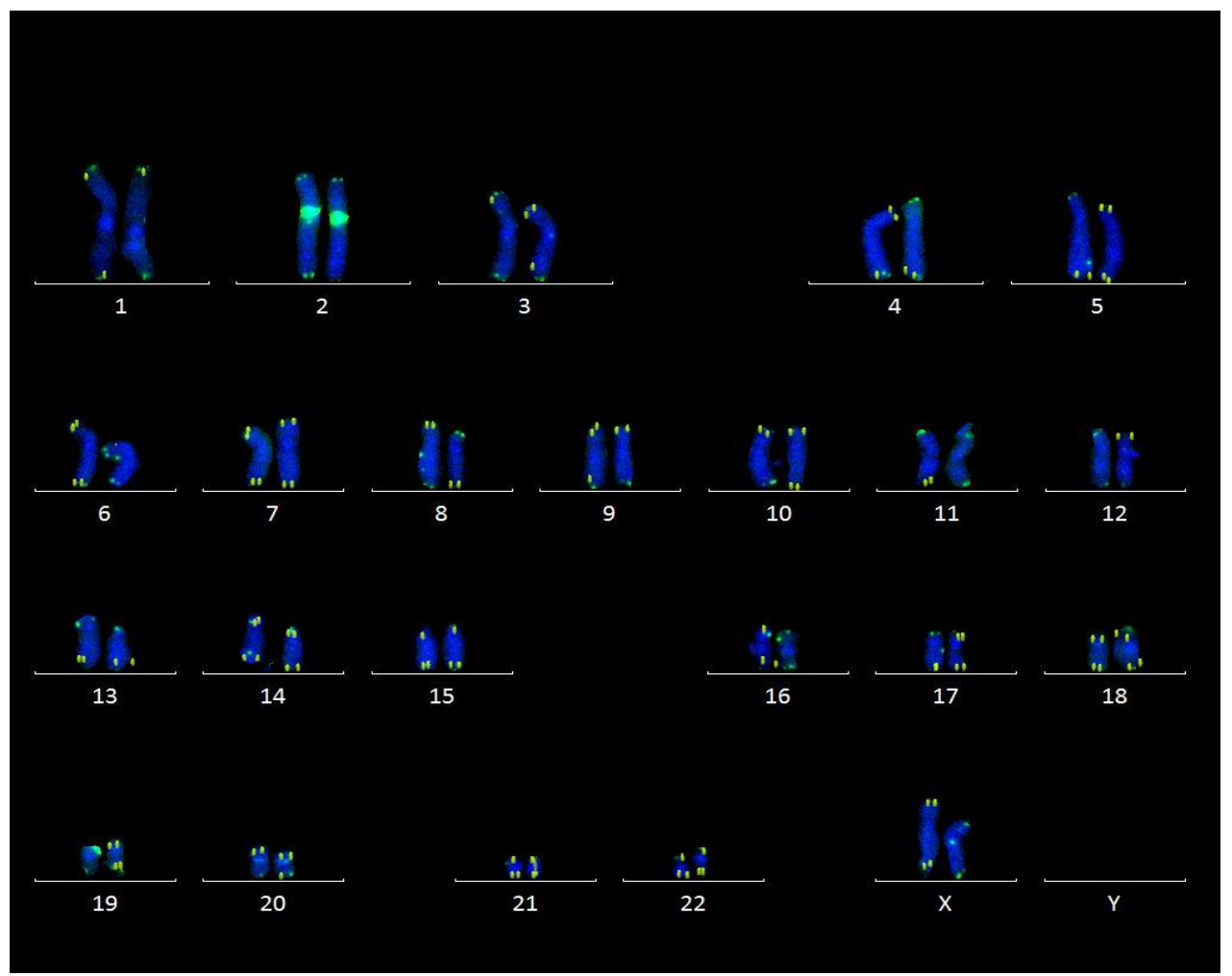

Figure (1): Image of a karyogram showing probe fluorescence at each telomere and the centromere reference probe on chromosome 2 using PNA FISH probes

\section{DISCUSSION}

Short telomeres have been found as a marker for age-related diseases such as dementia (Thomas et al., 2008). Telomere length was found to be significantly $(\mathrm{P}<0.05)$ reduced in AD compared with the control. 
A significant correlation between mean telomere length in AD patients and telomere length in healthy cases was observed.

Numerous studies on human populations reported that telomere length decreases with AD (Grodstein et al., 2008). Changes of blood cell telomere length might not reflect changes of brain cell telomere length. It has been reported that patients with $\mathrm{AD}$ exhibit an accelerated telomere loss in peripheral blood cells (Devore et al., 2011).

In our study it was found that the telomeric length is inversely proportional with Alzheimer disease period; so, it was supposed that the disease condition may provide a detrimental context for telomere length maintaining.

Smoking is one of the main pollutants causing oxidative stress and inflammatory conditions. Exposure to such stresses results in telomeric attrition (Révész et al., 2014).

Compared to genomic DNA, telomeric DNA lacks an efficient repair mechanism for single-strand breaks produced by reactive oxygen species, particularly the hydroxyl radical, compared with the genomic DNA. Telomere length is associated with smoking in $\mathrm{AD}$ patients. Another study clarifies the causes of accelerated telomere shortening in AD patients (Takata et al., 2012) which consisted with our findings.

In the current study, smokers, non-smokers ratio was $1: 1$. In the smoker group of $\mathrm{AD}$ patients (mean: $4.88 \mathrm{~kb}, \mathrm{SD}: 1.96$ ) in telomeric length, and non- 
smokers have mean $5.72 \pm$ SD 2.13, respectively which illcuidate effects of smoking on the AD smokers patients telomeres than non-smokers.

Q-FISH technique has an advantage over other telomere length methodologies; it enables the analysis of all telomere lengths from each individual chromosome in a population of metaphase-induced cells where simultaneous karyotyping can be performed. This allows the identification of specific telomeres on specific chromosomes as potential markers of an adverse effect.

A disadvantage of Q-FISH is quite labour-intensive and is generally not suitable for high throughput analysis. The technique depends on wellprepared metaphase cells, and it is vital that the equipment and samples are adjusted /normalized correctly in order for the quantification be accurate. Furthermore, while only a small quantity of cells is needed, it is difficult to get a sufficient number in metaphase at once.

A significant correlation has been found between $\mathrm{AD}$ and telomere length shortening. The environmental influence of smoking had an additional effect on telomere shortening in $\mathrm{AD}$.

\section{REFERENCES}

Barber, R. C. (2012): The genetics of Alzheimer's disease. Scientifica, 2012.

Bertram, L.; McQueen, M. B.; Mullin, K.; Blacker, D. and Tanzi, R. E. (2007): Systematic meta-analyses of Alzheimer disease genetic association studies: the AlzGene database. Nature genetics, 39(1), 17-23. 
Bertram, L. and Tanzi, R. E. (2012): The genetics of Alzheimer's disease. In Progress in molecular biology and translational science (Vol. 107, pp. 79-100). Academic Press.

Bird, T. D. (2008): Genetic aspects of Alzheimer disease. Genetics in Medicine, 10(4), 231-239.

Corder, E. H.; Saunders, A. M.; Strittmatter, W. J.; Schmechel, D. E.; Gaskell, P. C.; Small, G. and Pericak-Vance, M. A. (1993): Gene dose of apolipoprotein E type 4 allele and the risk of Alzheimer's disease in late onset families. Science, 261(5123), 921-923.

Crous-Bou, M.; Minguillón, C.; Gramunt, N. and Molinuevo, J. L. (2017): Alzheimer's disease prevention: from risk factors to early intervention. Alzheimer's Research \& Therapy, 9(1), 71.

Devore, E. E.; Prescott, J.; De Vivo, I. and Grodstein, F. (2011): Relative telomere length and cognitive decline in the Nurses' Health Study. Neuroscience letters, 492(1), 15-18.

Grodstein, F.; Van Oijen, M.; Irizarry, M. C.; Rosas, H. D.; Hyman, B. T.; Growdon, J. H. and De Vivo, I. (2008): Shorter telomeres may mark early risk of dementia: preliminary analysis of 62 participants from the nurses' health study. PloS one, 3(2), e1590.

Heidinger, B. J.; Blount, J. D.; Boner, W.; Griffiths, K.; Metcalfe, N. B. and Monaghan, P. (2012): Telomere length in early life predicts lifespan. Proceedings of the National Academy of Sciences, 109(5), 1743-1748.

Iourov, I. Y. (2017): Quantitative fluorescence in situ hybridization (QFISH). In: Cancer Cytogenetics (pp. 143-149). Humana Press, New York, NY.

Jenkins, F. J.; Kerr, C. M.; Fouquerel, E.; Bovbjerg, D. H. and Opresko, P. L. (2017): Modified terminal restriction fragment analysis for quantifying telomere length using in-gel hybridization. JoVE (Journal of Visualized Experiments), (125), e56001. 
Lai, T. P.; Wright, W. E. and Shay, J. W. (2018): Comparison of telomere length measurement methods. Philosophical Transactions of the Royal Society B: Biological Sciences, 373(1741), 20160451.

Moulton, P. V. and Yang, W. (2012): Air pollution, oxidative stress and Alzheimer's disease. Journal of Environmental and Public Health, 2012.

Muraki, K.; Nyhan, K.; Han, L. and Murnane, J. P. (2012): Mechanisms of telomere loss and their consequences for chromosome instability. Frontiers in oncology, 2, 135.

Passos, J. F.; Saretzki, G. and Von Zglinicki, T. (2007): DNA damage in telomeres and mitochondria during cellular senescence: Is there a connection?. Nucleic Acids Research, 35(22), 7505-7513.

Perner, S.; Brüderlein, S.; Hasel, C.; Waibel, I.; Holdenried, A.; Ciloglu, N. and Möller, P. (2003): Quantifying telomere lengths of human individual chromosome arms by centromere-calibrated fluorescence in situ hybridization and digital imaging. The American Journal of Pathology, 163(5), 1751-1756.

Pray, L.; Forum, F. and Board, N. (2015): Relationships among the brain, the digestive system, and eating behavior: Workshop summary. National Academies Press.

Reichert, S. and Reichert, S. (2014): Determinants of telomere length and implications in life history trade-offs. (Doctoral dissertation, Université de Strasbourg).

Révész, D.; Verhoeven, J. E.; Milaneschi, Y.; de Geus, E. J.; Wolkowitz, O. M. and Penninx, B. W. (2014): Dysregulated physiological stress systems and accelerated cellular aging. Neurobiology of aging, 35(6), 1422-1430.

Serrano-Pozo, A.; Frosch, M. P.; Masliah, E. and Hyman, B. T. (2011): Neuropathological alterations in Alzheimer disease. Cold Spring Harbor Perspectives in Medicine, 1(1), a006189. 
Shalev, I. (2012): Early life stress and telomere length: investigating the connection and possible mechanisms: a critical survey of the evidence base, research methodology and basic biology. Bioessays, 34(11), 943-952.

Takata, Y.; Kikukawa, M.; Hanyu, H.; Koyama, S.; Shimizu, S.; Umahara, T. and Ohyashiki, J. H. (2012): Association between ApoE phenotypes and telomere erosion in Alzheimer's disease. Journals of Gerontology Series A: Biomedical Sciences and Medical Sciences, 67(4), 330-335.

Thomas, P.; O'Callaghan, N. J. and Fenech, M. (2008): Telomere length in white blood cells, buccal cells and brain tissue and its variation with ageing and Alzheimer's disease. Mechanisms of Ageing and Development, 129(4), 183-190.

Ungar, L.; Altmann, A. and Greicius, M. D. (2014): Apolipoprotein E, gender, and Alzheimer's disease: an overlooked, but potent and promising interaction. Brain imaging and behavior, 8(2), 262-273.

Van der Vaart, H.; Postma, D. S.; Timens, W. and Ten Hacken, N. H. (2004): Acute effects of cigarette smoke on inflammation and oxidative stress: a review. Thorax, 59(8), 713-721.

Verma, R. S. and Babu, A. (1995): Human chromosomes: principles and techniques. McGraw-Hill.

Wu, L.; Rosa-Neto, P.; Hsiung, G. Y. R.; Sadovnick, A. D.; Masellis, M.; Black, S. E. and Gauthier, S. (2012); Early-onset familial Alzheimer's disease (EOFAD). Canadian Journal of Neurological Sciences, 39(4), 436-445. 


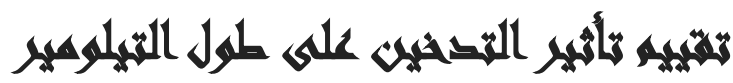

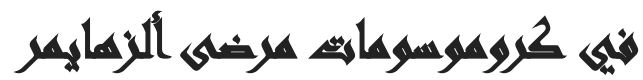

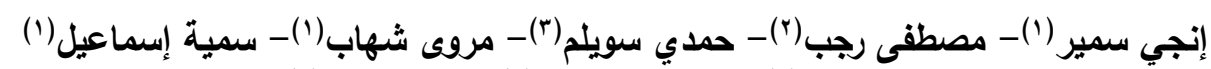

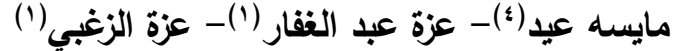

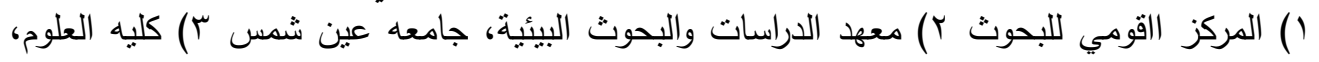

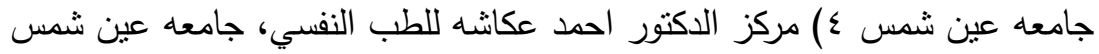

\section{المستخلي}

المقدمه: مرض ألزهايمر هو مرض عصبي وراثي خطير بيتأثر المرضى به بعوامل بيئيَه كثثرة وهو

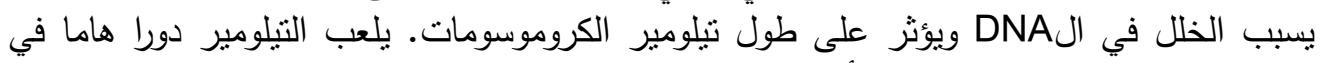

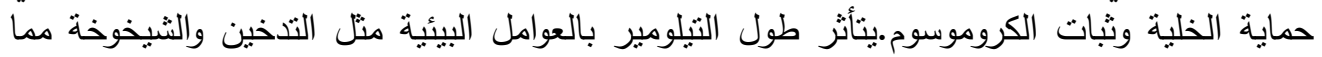

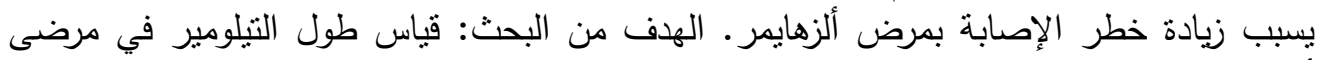

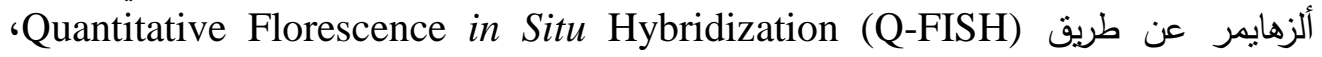

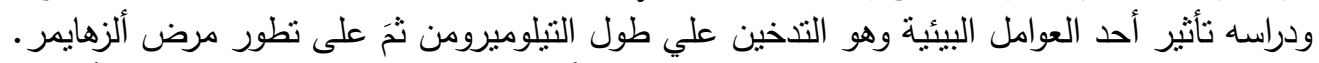

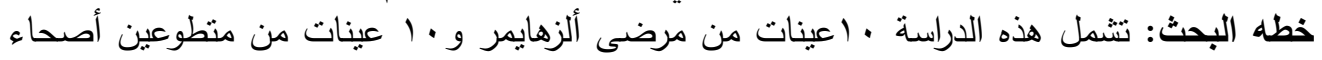

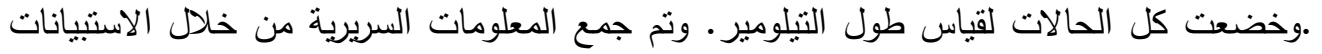
المصممه لذلك. نتائج البحث: أثتتت هذه الدراسة أن هناك علاقة بين قصر طول التيلومير ومرض ألزهايمر.

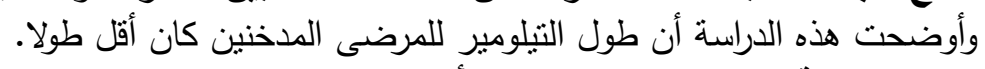

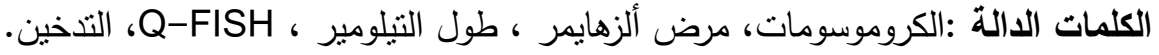

